\section{Research Square}

Preprints are preliminary reports that have not undergone peer review. They should not be considered conclusive, used to inform clinical practice, or referenced by the media as validated information.

\title{
Complete genome sequence of Arthrobacter sp. PAMC25564 and comparative genome analysis for elucidating the role of CAZymes in cold adaptation
}

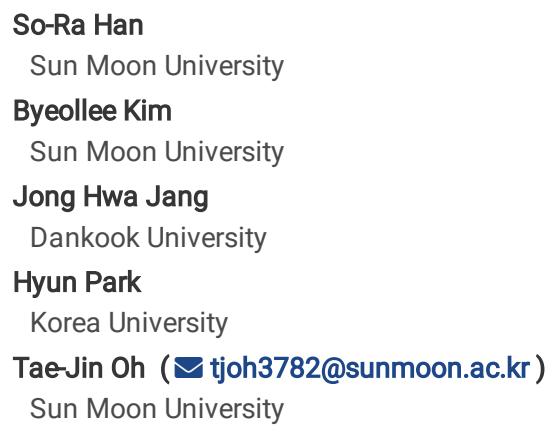

\section{Research Article}

Keywords: Arthrobacter species, CAZyme, cold-adapted bacteria, genetic patterns, glycogen metabolism, trehalose pathway

Posted Date: December 16th, 2020

DOI: https://doi.org/10.21203/rs.3.rs-118769/v1

License: (a) (1) This work is licensed under a Creative Commons Attribution 4.0 International License. Read Full License 


\section{Abstract}

Background: The Arthrobacter group is a known isolate from cold areas, the species of which are highly likely to play diverse roles in low temperatures. However, their role and survival mechanisms in cold regions such as Antarctica are not yet fully understood. In this study, we compared the genomes of sixteen strains within the Arthrobacter group, including strain PAMC25564, to identify genomic features that adapt and survive life in the cold environment.

Results: The genome of Arthrobactersp. PAMC25564 comprised 4,170,970 bp with $66.74 \%$ GC content, a predicted genomic island, and 3,829 genes. This study provides an insight into the redundancy of CAZymes for potential cold adaptation and suggests that the isolate has glycogen, trehalose, and maltodextrin pathways associated to CAZyme genes. This strain can utilize polysaccharide or carbohydrate degradation as a source of energy. Moreover, this study provides a foundation on which to understand how the Arthrobacter strain produces energy in an extreme environment, and the genetic pattern analysis of CAZymes in cold-adapted bacteria can help to determine how bacteria adapt and survive in such environments.

Conclusions: We characterized the Arthrobacter sp. PAMC25564 complete genome and comparative analysis, provided an insight into the redundancy of CAZymes for potential cold adaptation. This provide a foundation to understand how Arthrobacter strain produces energy in an extreme environment, there are reports on the use of CAZymes in cold environments. Therefore, we suppose that this process has allowed Arthrobacter species to establish a symbiotic relationship with other bacteria in cold environments or live independently thanks to their capacity for adapting to environmental changes.

\section{Background}

The Arthrobacter genus is member of the family Micrococcaceae, which belongs to the phylum Actinobacteria [1, 2]. Arthrobacter species are often isolated from soil, where they contribute to biochemical cycles and decontamination [3]. These species have been isolated worldwide from a variety of environments, including sediments [4], human clinical specimens [5], water [6] glacier cryoconite [7], sewage [8], and glacier ice [9]. Cold environments are present in about $75 \%$ of the Earth, and their study provides information about new microorganisms and the evolution of cold environments [10]. Psychrophilic microorganisms have colonized all permanently cold environments, from the deep sea to mountains and polar regions [11]. Cold-adapted microorganisms utilize a wide range of metabolic strategies to grow in diverse environments. In general, the ability to adapt to low temperatures requires the microorganisms to sense a decrease in temperature, which induces the upregulation of cold-associated genes $[12,13]$. Therefore, these kinds of enzymes have been studied for everyday life applications such as detergent additives; textile industry; food industry; and bioremediation [14].

CAZymes are enzymes associated with the biosynthesis, binding, and catabolism of carbohydrates. These enzymes are divided into several groups based on their catalytic activity: glycoside hydrolase (GH); carbohydrate esterase (CE); polysaccharide lyase (PL); glycosyltransferase (GT); and auxiliary activities (AA). CAZymes may have non-catalytic subunits. Carbohydrates are catabolized in a carbohydrate-binding module (CBM). CAZymes are well known in biotechnology, and their industrial applications are of interest to many researchers because CAZymes produce precursors for bio-based products such as food, paper, textile, animal feed, and other chemicals, including biofuels $[15,16]$.

Most bacteria can use glycogen as an energy storage compound, and the enzymes involved in its metabolism are well known. A recent study showed the physiological impact of glycogen metabolism in the survival of bacteria living in extreme environments [17]. Some microorganisms can adapt quickly to continuously changing environmental conditions, by accumulating energy storage compounds to cope with transient starvation periods. These strategies use glycogen-like structures such as a polysaccharides composed of $a^{-}{ }^{-}$-glycosyl units connected by $a-1,4$ linkages and branched by $a-1,6$ glycosidic linkages. These biopolymers differ in their chain length and branching occurrence. To be used as carbon and energy sources, their glucose units are released by specific enzymes [18].

Microorganisms have synergistic enzymes capable of decomposing plant cell walls to release glucose. Therefore, this phenomenon can be used as energy supply to maintain microbial growth [19]. Starch is an excellent source of carbon and energy for microbes that produce proteins responsible for the extracellular hydrolysis of starch, in-cell absorption of fructose, and further decomposition into glucose [20]. In addition, strains that metabolize glycogen show important physiological functions, including the use of energy storage compounds for glycogen metabolism. These pathways act as carbon pools that regulate carbon fluxes [21], and part of this capability are due to CAZymes. The genetic patterns of CAZymes in cold-adapted bacteria can help to understand how survival adaptation can be achieved in extreme low temperature environments.

\section{Results And Discussion}

\section{Profile of the complete genome of Arthrobacter sp. PAMC25564}

As shown in Table 1, the complete genome of $A$. sp. PAMC25564 is composed of a circular chromosome of 4,170,970 bp with a $66.74 \%$ GC content. The chromosome is predicted to include 3,829 genes, from which 3,613 protein-encoding genes were functionally assigned, whereas the remaining genes were predicted as hypothetical proteins. We annotated 147 pseudogenes, 15 rRNA genes, and 51 tRNA genes distributed through the genome. From the predicted genes, $3,449(90.08 \%)$ were classified into 20 functional COG categories, whereas the remaining $380(9.92 \%)$ remained un-classified. The most numerous COG categories were S genes with unknown function (705 genes), K (298 genes), E (280 genes), G (276 genes), and C (259 genes) (Fig. 1). Many of these genes are related to amino acid transport, transcription, carbohydrate transport, and energy production/conversion, which suggests that this strain utilizes CAZymes for energy storage and carbohydrate metabolism. Most bacteria rely on cell respiration to catabolize carbohydrates to obtain the energy used during photosynthesis for converting carbon dioxide into carbohydrates. The energy is stored temporarily in the form of high-energy molecules such as ATP and used in several cell processes [33,34]. Therefore, we predicted that the PAMC25564 strain could utilize carbohydrate degradation to obtain energy. 
Table 1

Genome features of Arthrobactersp.

PAMC25564.

\begin{tabular}{|ll|}
\hline Feature & Value \\
\hline A; Genome Statistics & \\
\hline Contigs & 1 \\
\hline Total length bp; & $4,170,970$ \\
\hline N50 & $4,170,970$ \\
\hline L50 & 1 \\
\hline GC \%; & 66.74 \\
\hline B; Genome features & \\
\hline Assembly level & Complete genome \\
\hline Chromosome genes & 3,829 \\
\hline Protein coding genes & 3,613 \\
\hline Pseudogenes & 147 \\
\hline rRNA genes & 15 \\
\hline tRNA genes & 51 \\
\hline
\end{tabular}

\section{S rRNA phylogenetic analysis and ANI values}

The identification of $A$. sp. PAMC25564 was verified using 16S rRNA sequence analysis (Fig. 2). This strain is phylogenetically placed among Arthrobacter and Pseudarthrobacter species. The results from phylogenetic analysis, BLAST analysis, and EzBio Cloud analysis revealed closely related strains such as $P$. sulfonivirans ALL (T) (99.09\%), P. siccitolerans 4J27 (T) (98.48\%), A. ginsengisoli DCY81 (T) (98.23\%), and P. phenanthrenivorans Sphe3 (T) (98.13\%). These results confirmed that isolate PAMC25564 belongs to the family Micrococcaceae, phylum Actinobacteria. Recently, several Arthrobacter species have been reclassified into new genera, based on 16S rRNA sequence similarities and chemotaxonomic traits such as peptidoglycan types, quinone systems, and/or polar lipid profiles [35]. Therefore, it has been proposed to reclassify within the genus Arthrobactermembers of these five genera: Paenarthrobacter gen. nov., Pseudarthrobacter gen. nov., Glutamicibactergen. nov., Paeniglutamicibacter gen. nov., and Pseudoglutamicibacter gen. nov. Among them,

Pseudarthrobacter group would be reclassified into the genus Arthrobacter as: $A$. chlorophenolicus, $A$. defluvii, A. equi, A. niigatensis, A. oxydans, $A$. phenanthrenivorans, A. polychromogenes, A. scleromae, A. siccitolerans, and A. sulfonivorans [36]. Therefore, the PAMC25564 strain will probably be reclassified into the genus Pseudarthrobacter. As shown in Fig. 3, each ANI values ranged from 70.67 to $98.46 \%$. So, we were confirmed that comparative genome results much lower than the common ANI values of $92-94 \%$. In general, bacterial comparative genome analysis uses this method. The ANI analysis shows the average nucleotide identity of all bacterial orthologous genes that are shared between any two genomes and offers a robust resolution between bacterial strains of the same or closely related species (i.e., species showing $80-100 \%$ ANI) [37]. However, ANI values do not represent genome evolution, because orthologous genes can widely vary between the genomes being compared. Nevertheless, ANI closely reflects the traditional microbiological concept of DNA-DNA hybridization relatedness for defining species, so many researchers used this method, since it takes into account the fluid nature of bacterial gene pool and hence implicitly considers shared functions [38]. So, this mean the PAMC25564 strain could either belong to the species from which Arthrobacter diverged, or this could be a Pseudarthrobacter closely related new species.

\section{CAZymeencoding genes in Arthrobacter sp. PAMC25564}

Among the 3,613 identified protein-encoding genes in PAMC25564, 108 were significantly annotated and classified into CAZyme groups: GH, GT, CE, AA, $\mathrm{CBM}$, and PL using dbCAN2. The results provided an insight into the carbohydrate utilization mechanisms of PAMC25564. The signal P analysis predicted that 11 genes contained signal peptides. We found that proteins were distributed as follows: $33 \mathrm{GHs}, 45 \mathrm{GTs}, 23 \mathrm{CEs}, 5$ AAs, and 2 CBMs. However, no protein was assigned to the PL group. Most annotation results of GH genes revealed that the PAMC25564 genome has genes involved in glycogen and trehalose metabolism pathways such as $\beta$-glucosidase (GH1), glycogen debranching proteins (CBM48 and GH13_11), (1 $\rightarrow 4$ )-a-D-glucan 1-a-D-glucosylmutase (GH13_26), a-glucosyltransferase (GH13), a-trehalose phosphorylase (GH65), and 4-a-glucanotransferase (GH77) (Table 2). Previous studies showed the complex interplay of glycogen metabolism in colony development of Streptomycetes (in Actinomycetes species was only reported), showing that spore germination is followed by an increase in glycogen metabolism [39]. The underlying genetic and physiological mechanisms of spore germination remain unknown, but some mechanisms associated with the accumulation of nutrients as biomass and storage materials in the substrate mycelium during morphological phases of development have been reported [40]. Recently, Shigella sp. PAMC 28760, of pathogens isolated from Antarctica, was also reported to be able to adapt and survive in cold environments through glycogen metabolism [64]. Nonetheless, glycogen metabolism in bacteria remains unknown, even though it has been well-studied in eukaryotes [41]. However, we could predict the specificity of PAMC25564 strain genes involved in glycogen and trehalose metabolism. 
List of CAZyme GH enzymes from Arthrobacter sp. PAMC25564.

\begin{tabular}{|c|c|c|c|c|}
\hline $\begin{array}{l}\text { CAZyme } \\
\text { group }\end{array}$ & Enzyme activity & Gene position & $\begin{array}{l}\text { EC } \\
\text { number }\end{array}$ & Number \\
\hline \multirow[t]{2}{*}{$\mathrm{GH} 1$} & \multirow[t]{2}{*}{$\beta$-Glucosidase } & 1206507_1208054 & \multirow[t]{2}{*}{ EC 3.2.1.21 } & \multirow[t]{2}{*}{2} \\
\hline & & 1548357_1546930 & & \\
\hline $\mathrm{GH} 2$ & $\beta$-Glucuronidase & 230633_228825 & EC 3.2.1.31 & 1 \\
\hline \multirow[t]{2}{*}{$\mathrm{GH} 3$} & \multirow[t]{2}{*}{$\beta$-Glycosyl hydrolase } & 226049_223737 & \multirow[t]{2}{*}{-} & \multirow[t]{2}{*}{2} \\
\hline & & 1615559_1617091 & & \\
\hline $\mathrm{GH} 4$ & 6-Phospho- $\beta$-glucosidase & 1608453_1606978 & EC 3.2.1.86 & 1 \\
\hline \multirow[t]{13}{*}{ GH13 } & Malto-oligosyltrehalose/ & 1599543_1601333 & EC 3.2.1.141 & \multirow[t]{13}{*}{8} \\
\hline & trehalohydrolaseGH13_10; & & & \\
\hline & Limit dextrin a-1,6-maltotetraose-hydrolase GH13_11; & 4158486_4156372 & EC 3.2.1.196 & \\
\hline & Trehalose synthase & 4150667_4148871 & EC 5.4.99.16 & \\
\hline & a-Amylase GH13_16; & & EC 3.2.1.1 & \\
\hline & Malto-oligosyltrehalose synthase GH13_26; & 1597186_1599498 & EC 5.4.99.15 & \\
\hline & Glucanase glge & $4152718 \_4150673$ & EC 3.2.1.- & \\
\hline & GH13_3; & & & \\
\hline & a-Glucosidase & 1490553_1492259 & EC 3.2.1.20 & \\
\hline & GH13_30; & & & \\
\hline & Limit dextrin a-1,6-maltotetraose-hydrolase & 1594748_1597189 & EC 3.2.1.196 & \\
\hline & CBM48 + GH13_11; & & & \\
\hline & 1,4-a-Glucan glycogen; branching enzyme CBM48 + GH13_9; & 4148869_4145174 & EC 2.4.1.18 & \\
\hline \multirow[t]{4}{*}{ GH15 } & \multirow[t]{3}{*}{ Glucoamylase } & 2725735_2726796 & \multirow[t]{3}{*}{ EC 3.2.1.3 } & \multirow[t]{4}{*}{4} \\
\hline & & 1210063_1211832 & & \\
\hline & & 2262201_2264033 & & \\
\hline & Trehalose-6-phosphate phosphatase & 943180_945807 & EC 3.1.3.12 & \\
\hline \multirow[t]{2}{*}{$\mathrm{GH} 23$} & Peptidoglycan-binding lysm & 3355397_3356797 & - & \multirow[t]{2}{*}{2} \\
\hline & Membrane-bound lytic murein transglycosylase & 3043759_3043151 & EC 4.2.2.- & \\
\hline $\mathrm{GH} 25$ & $1,4-\beta-N-A c e t y l m u r a m i d a s e$ & 1863081_1865612 & EC 3.2.1.92 - & 1 \\
\hline $\mathrm{GH} 30$ & Endo-1,6- $\beta$-galactosidase & 731722_733167 & EC 3.2.1.164 & 1 \\
\hline \multirow[t]{2}{*}{ GH32 } & Sucrose-6-phosphate hydrolase & 3442058_3440550 & EC 3.2.1.26 & \multirow[t]{2}{*}{2} \\
\hline & $\beta$-Fructosidase & 1383596_1384843 & EC 3.2.1.26 & \\
\hline GH33 & Sialidase & 613011_614603 & EC 3.2.1.18 & 1 \\
\hline GH38 & a-Mannosidase & 4082745_4079713 & EC 3.2.1.24 & 1 \\
\hline GH53 & Galactosidase & 2955779_2956930 & - & 1 \\
\hline \multirow[t]{3}{*}{ GH65 } & Maltose phosphorylase/ & 392560_390227 & EC 2.4.1.8 & 2 \\
\hline & Trehalose phosphorylase & & EC 2.4.1.64 & \\
\hline & Trehalose-6-phosphate phosphatase & 342376_339176 & EC 3.1.3.12 & \\
\hline GH76 & Fructose-bisphosphate aldolase & 137645_138862 & EC 4.1.2.13 & 1 \\
\hline GH77 & 4-a-Glucanotransferase amylomaltase; & 2730522_2728363 & EC 2.4.1.25 & 1 \\
\hline \multirow[t]{2}{*}{ GH109 } & \multirow[t]{2}{*}{ Gluconokinase } & 787401_788513 & \multirow[t]{2}{*}{ EC 2.7.1.12 } & \multirow[t]{2}{*}{2} \\
\hline & & 236269_235103 & & \\
\hline
\end{tabular}


We compared CAZyme genes from Arthrobacter species to speculate about their bacterial lifestyles and identified relevant CAZymes for potential applications in biotechnology. Considering the accessibility of available genome data, the complete genomes of 26 strains were chosen for the comparative analysis of CAZymes: 19 genomes of Arthrobacter spp., 1 genome of A. crystallopoietes, 3 genomes of A. alpinus, and 3 genomes of Pseudarthrobacter spp. (Table 3). Our results showed that the number of genes encoding glycogen and trehalose metabolism-associated CAZymes ranged from a minimum of 56 (A. sp. YC-RL1) to a maximum of 177 (A. sp. YN). We predicted that genes such as CE14, CE9, GH23, GH65, GT2, GT20, GT28, GT39, GT4, and GT51 were in each of the 26 genomes. In addition, GH13, GH65, GH77, GT5, and GT20 (glycogen and trehalose-related genes) are involved in energy storage. These genes are involved in glycogen degradation and trehalose pathways and were found in strains PAMC25564, 24S4-2, FB24, Hiyo8, KBS0702, MN05-02, PGP41, QXT$31, \mathrm{U} 41$, UKPF54-2, A6, Ar51, and sphe3. These genes code for proteins with a strong ability to store and release energy. We found that strain PAMC25564 had the largest number of CAZyme genes. In general, CAZymes are large group of protein and this is mainly responsible for the degradation and biosynthesis/modification of polysaccharide but not all the members of this group are secreted proteins. This confirmed the little difference through results (Fig. 4).

Table 3

Genome information and comparative data of CAZymes from 26 strains including Arthrobacter sp. PAMC25564.

\begin{tabular}{|c|c|c|c|c|c|c|c|c|c|c|}
\hline Species & Strain & $\begin{array}{l}\text { Size } \\
\text { Mb; }\end{array}$ & GC \%; & Replicons & Plasmid & Gene & Protein & tRNAs & rRNAs & References \\
\hline \multirow[t]{19}{*}{ Arthrobactersp. } & PAMC25564 & 4.17097 & 66.70 & NZ_CP039290.1 & 0 & 3,829 & 3,613 & 51 & 15 & This study \\
\hline & $24 S 4-2$ & 5.56375 & 65.10 & NZ_CP040018.1 & 0 & 5,152 & 4,522 & 50 & 15 & Unpublished \\
\hline & YN & 5.06355 & 62.70 & NZ_CP022436.1 & 0 & 4,673 & 4,387 & 55 & 18 & Unpublished \\
\hline & QXT-31 & 5.04157 & 66.00 & NZ_CP019304.1 & 0 & 4,593 & 4,379 & 54 & 18 & Unpublished \\
\hline & Rue61a & 5.08104 & 62.23 & NC_018531.1/CP003203.1 & 2 & 4,693 & 4,568 & 53 & 18 & {$[55]$} \\
\hline & FB24 & 5.07048 & 65.42 & NC_008541.1/CP000454.1 & 3 & 4,623 & 4,486 & 51 & 15 & {$[56]$} \\
\hline & PAMC25486 & 4.59358 & 62.80 & NZ_CP007595.1 & 0 & 4,154 & 3,995 & 53 & 18 & Unpublished \\
\hline & $Z X Y-2$ & 5.05871 & 63.35 & NZ_CP017421.1 & 5 & 4,700 & 4,505 & 54 & 18 & Unpublished \\
\hline & U41 & 4.79263 & 66.38 & NZ_CP015732.1 & 3 & 4,407 & 4,134 & 51 & 15 & Unpublished \\
\hline & DCT-5 & 4.53075 & 66.22 & NZ_CP029642.1 & 1 & 4,040 & 3,816 & 50 & 15 & Unpublished \\
\hline & PGP41 & 4.27024 & 65.40 & NZ_CP026514.1 & 0 & 3,917 & 3,760 & 49 & 12 & Unpublished \\
\hline & ERGS1:01 & 4.93669 & 65.41 & NZ_CP012479.1 & 2 & 4,481 & 4,232 & 41 & 6 & [57] \\
\hline & YC-RL1 & 4.01864 & 64.04 & NZ_CP013297.1 & 2 & 3,754 & 3,606 & 66 & 19 & [58] \\
\hline & Hiyo4 & 3.77925 & 65.00 & AP014718.1 & 0 & 5,182 & 5,120 & 50 & 12 & [59] \\
\hline & KBS0702 & 3.64955 & 67.90 & NZ_CP042172.1 & 0 & 3,373 & 3,243 & 51 & 15 & Unpublished \\
\hline & UKPF54-2 & 3.51782 & 68.50 & NZ_CP040174.1 & 0 & 3,238 & 3,110 & 50 & 15 & Unpublished \\
\hline & MN05-02 & 3.64342 & 68.81 & AP018697.1 & 1 & 3,608 & 3,543 & 52 & 12 & Unpublished \\
\hline & Hiyo8 & 5.02672 & 63.76 & AP014719.1 & 2 & 7,108 & 7,038 & 53 & 15 & [59] \\
\hline & ATCC21022 & 4.43490 & 63.40 & NZ_CP014196.1 & 0 & 4,078 & 3,910 & 53 & 12 & {$[60]$} \\
\hline $\begin{array}{l}\text { Arthrobacter } \\
\text { crystallopoietes }\end{array}$ & DSM 20117 & 5.03270 & 64.36 & NZ_CP018863.1 & 2 & 4,634 & 4,425 & 48 & 15 & Unpublished \\
\hline \multirow{3}{*}{$\begin{array}{l}\text { Arthrobacter } \\
\text { alpinus }\end{array}$} & R3.8 & 4.04645 & 62.20 & NZ_CP012677.1 & 0 & 3,732 & 3,523 & 51 & 18 & Unpublished \\
\hline & ERGS4:06 & 4.33365 & 60.59 & NZ_CP013200.1 & 1 & 3,850 & 3,581 & 53 & 25 & {$[61]$} \\
\hline & A3 & 4.45829 & 60.64 & NZ_CP013745.1 & 1 & 4,033 & 3,902 & 52 & 19 & Unpublished \\
\hline $\begin{array}{l}\text { Pseudarthrobacter } \\
\text { phenanthrenivorans }\end{array}$ & Sphe3 & 4.53532 & 65.38 & NC_015145.1/CP002379.1 & 2 & 4,278 & 4,052 & 50 & 12 & {$[62]$} \\
\hline $\begin{array}{l}\text { Pseudarthrobacter } \\
\text { chlorophenolicus }\end{array}$ & A6 & 4.98087 & 65.98 & NC_011886.1/CP001341.1 & 2 & 4,685 & 4,505 & 49 & 15 & {$[63]$} \\
\hline $\begin{array}{l}\text { Pseudarthrobacter } \\
\text { sulfonivorans }\end{array}$ & Ar51 & 5.04376 & 64.70 & NZ_CP013747.1 & 1 & 4,640 & 4,408 & 50 & 12 & Unpublished \\
\hline
\end{tabular}


Glycogen is an energy source for plants, animals, and bacteria and is one of the most common carbohydrates. Glycogen consists of D-glucose residues joined by a $(1 \rightarrow 4)$ links; and it is a structural part of cellulose and dextran [42]. Glycogen is a polymer with approximately $95 \%$ of a- 1,4 linkages, and $5 \%$ of a-1, 6 branching linkages. In bacteria, glycogen metabolism includes five essential enzymes: ADP-glucose pyrophosphorylase (GlgC), glycogen synthase (GlgA), glycogen branching enzyme (GlgB), glycogen phosphorylase (GlgP), and glycogen debranching enzyme (GlgX) [43]. To adapt and survive in a cold environment, organisms need well-developed functional energy storage systems, one of which is glycogen synthesis. Bacteria have a passive energy saving strategy to adapt to cold environmental conditions such as nutrient deprivation, by using a slow glycogen degradation. Glycogen has the hypothesis of durability energy reserves, which have been reported as a Durable Energy Storage Mechanism (DESM) to account for the long-term survival of some bacteria in cold environments [44]. Metabolism of maltodextrin has been linked with osmoregulation and sensitivity of bacterial endogenous induction to hyperosmolarity, which is related to glycogen metabolism. Glycogen-generated maltotetraose is dynamically metabolized by maltodextrin phosphorylase (MalP) and maltodextrin glucosidase (MalZ), while 4-a-glucanotransferase (MalQ) is responsible for maltose recycling to maltodextrins [45]. Maltotetraose is produced using GlgB, MalZ, MalQ, and glucokinase (Glk), which act on maltodextrin and glucose. On the other hand, glucose-1-phosphate can be formed by MalP for glycogen synthesis or glycolysis [46]. Thus, glycogen degradation can play an essential role in bacterial adaptation to the environment. Additionally, maltose may form capsular a-glucan, which plays a role in environmental adaptation through the (TreS)-Pep2-GlgE-GlgB pathway [47, 48]. Previous studies indicate that trehalose is involved in bacterial adaptation to temperature fluctuation, hyperosmolarity, and desiccation resistance. Recently, the accumulation of trehalose and glycogen under cold conditions in Propionibacterium freudenreichii has been reported [49, 50]. Therefore, the role of glycogen in bacterial energy metabolism is closely linked to several metabolic pathways associated to bacterial persistence under environmental stresses such as starvation, drying, temperature fluctuations, and hyperosmolarity. Maltodextrin and trehalose pathways are examples of the relationship between glycogen and other metabolic pathways, as shown in Fig. 5. However, further exploration is needed to elucidate the relationship of glycogen with other compounds, and the mechanisms involved in bacterial persistence strategy [45]. The comparative analysis of predicted pathways for glycogen metabolism in Arthrobacter isolates (Additional file 1: Table S1), showed that in PAMC25564 the trehalose biosynthesis follows three metabolic pathways (OtsAB, TreYZ, and TreS) as in Mycobacterium [51]. The trehalose biosynthesis is well-known in numerous bacteria, for example, a defense strategy involving the accumulation of trehalose and three metabolic pathways to regulate osmotic stress is been reported in Corynebacterium g/utamicum [52]. These three metabolic pathways are used for producing trehalose in C. glutamicum, where the gene galU/ otsAB allows the increase of trehalose levels up to six times [53, 54]. This pathway was found in A. sp. PAMC25564, and it was predicted that such isolate could produce energy in cold environments.

\section{Glycogen metabolism and trehalose pathway in Arthrobacter species}

We investigated the glycogen metabolic pathways in each Arthrobacter strains (Fig. 6). To determine the 3 pathways of glycogen metabolism and trehalose pathway in Arthrobacter species, the level of dissimilarity was analyzed based on the composition of GH, GT, and other major enzymes from the 16 genomes. The analysis showed that only QXT-31, U41, and PGP41 shared with our strain the same genes and pathway, but other strains have a little different pattern. Therefore, we assumed that the PAMC25564 strain uses different pathways to obtain energy or degrade polysaccharides. Based on the above mentioned pathway-related genes, we confirmed that strains YN, Rue61, PAMC25486, ZXY-2, ERGS1:01, YC-RL1, ATCC21022, R.3.8, and A3 lack the malQ gene, which is responsible for maltose recycling to maltodextrins. Therefore, the energy supply may be compromised in such isolates. These Arthrobacter species showed a low number of genes for the three main pathways of trehalose. Therefore, we assumed that the PAMC25564 strain uses different pathways to obtain energy or degrade polysaccharides. Although most strains showed GalU/OtsAB genes, strains Hyo8 and ERGS1:01 lack the otsB gene (Fig. 6; Additional file 1: Table S1). So, we predicted that these strains would produce a significantly lower amount of trehalose, compared with the isolates having the OtsB gene. Additionally, we investigated the phosphotransferase system-related genes in strains R.3.8 and A3. These enzymes constitute another method used by bacteria for sugar uptake when the source of energy is phosphoenolpyruvate. As a result, these two strains were expected to produce polysaccharides by themselves or from an external source using phosphoenolpyruvate rather than consuming energy. Most of the compared strains were isolated from low temperature environments $\left(-18\right.$ to $\left.15^{\circ} \mathrm{C}\right)$, and most of them are known to adapt quickly to environmental changes. We expect that our results on glycogen metabolism, trehalose, and maltodextrin pathways will have a significant impact in industrial applications. The predicted trehalose metabolism in bacteria is important since they could be used for bioremediation. Additionally, these isolates would be an alternative for a cost-effective production of trehalose. We also predicted that trehalose metabolism varies among bacteria, depending on their metabolism and environmental conditions.

\section{Conclusions}

In this study, we elucidated the complete genome sequence of Arthrobacter sp. PAMC25564 and conducted a comparative genome analysis with other species for studying CAZyme patterns. We isolated bacteria from cryoconite under laboratory conditions and confirmed that the isolate is an Arthrobacter species, based on the analysis of 16S rRNA sequences. Although it has been reported the isolation of this species from extreme or contaminated environments, there are no reports on the use of CAZymes in cold environments. Therefore, we suppose that this process has allowed Arthrobacter species to establish a symbiotic relationship with other bacteria in cold environments, or live independently thanks to their capacity for adapting to environmental changes. We predicted that Arthrobactersp. PAMC25564 could produce energy by its own as fast as it can adapt to the environment. In summary, the PAMC25564 strain genome is $4.17 \mathrm{Mb}$ in size with a GC content of $66.74 \%$. The analysis of its complete genome suggested that the isolate has glycogen, trehalose, and maltodextrin pathways associated to CAZyme genes. We confirmed that PAMC25564 has 108 active CAZyme genes from the following groups, $5 \mathrm{AA}, 2 \mathrm{CBM}, 23 \mathrm{CE}, 33 \mathrm{GH}$, and $45 \mathrm{GT}$. In addition, a comparative genome analyses of Arthrobacter species revealed that they adapt quickly to the environment. In conclusion, we expect the genome sequence analysis provides valuable information regarding novel functional enzymes, especially CAZymes, which are active at low temperatures and can be used for biotechnological applications and fundamental research purposes. Additionally, this study provides a foundation to understand how the PAMC25564 strain produces energy in an extreme environment.

\section{Methods}


Arthrobactersp. PAMC25564 strain was isolated from the Cryoconite of Wurmkogel, Ötztaler Alps, Austria $\left(47^{\circ} 04^{\prime} \mathrm{N}, 12^{\circ} 41^{\prime} \mathrm{E}, 2820 \mathrm{~m}\right.$ to a maximum elevation) using 0.1 X R2A agar (MB cell Ltd., Seoul, Korea). The strain was isolated at an environmental temperature of $20^{\circ} \mathrm{C}$. The bacterial sample for DNA analysis was isolated at $15^{\circ} \mathrm{C}$ by using a pure R2A agar. DNA from A. sp. PAMC25564 was extracted using a QIAamp DNA Mini Kit (Qiagen Inc., Valencia, CA, USA). The quantity and purity of genomic DNA were determined using a spectrophotometer (Biochrome, Libra S35PC, UK). The extracted DNA was checked by agarose gel electrophoresis to evaluate its quality. DNA was stored at $-20{ }^{\circ} \mathrm{C}$ until use.

Genome sequencing and assembly of the whole genome of Arthrobacter sp. PAMC25564

Genome sequencing was performed using PacBio sequel single-molecule real-time (SMRT) sequencing technology (Pacific Biosciences, Menlo Park, CA, USA). SMRTbell library inserts ( $20 \mathrm{~kb}$ ) were sequenced using SMRT cells. Raw sequence data were generated from 77,075 reads and $821,081,934$ bp that were assembled de novo by using the hierarchical genome-assembly process (HGAP) protocol [22] and HGAP4 assembly using SMRT analysis software (ver. 2.3; Pacific Biosciences, https://github.com/PacificBiosciences/SMRT-Analysis). The complete genome sequence was deposited in the GenBank database under the GenBank accession number NZ_CP039290.1.

Genome annotation of Arthrobacter sp. PAMC25564

The PAMC25564 genome was annotated using the rapid annotation subsystem technology (RAST) server [23]. The predicted gene sequences were translated and searched in the National Center for Biotechnology Information (NCBI) non-redundant database, the Clusters of Orthologous Groups (COG) from the eggnog v.4.5.1 database [24], and the Kyoto Encyclopedia of Genes and Genomes (KEGG) database. A circular map of the PAMC25564 genome was prepared using the CGView comparison tool [25]. CAZyme gene analyses were carried out by running dbCAN tool [26] scans using hidden Markov model (HMM) profile downloaded from dbCAN2 HMMdb (version 7.0). The e-value cutoff was $1 \mathrm{e}-15$ and the coverage cutoff was $>0.35$. In addition, we used DIAMOND [27] (e-value < 1e102) and Hotpep [28] (frequency > 2.6, hits > 6) to improve the prediction accuracy.

\section{Phylogenetic analysis}

Strain PAMC25564 was compared with other Arthrobacter species on the base of 16S rRNA phylogenetic analysis. Alignments were performed using Basic Local Alignment Search Tool (BLAST) from the NCBI database and analyzed using EzBio Cloud (www.ezbiocloud.com). 16S rRNA sequences were aligned using MUSCLE $[29,30]$ and MEGA X [31] to reconstruct a neighbor-joining tree and maximum likelihood tree with 1000 bootstrap replications.

\section{Comparative genomics of Arthrobacter sp}

We used all complete genome sequences of Arthrobacter species available in GenBank (https://www.ncbi.nlm.nih.gov). Firstly, we determined the relationship of PAMC25564 with other strains from the same species using complete genome sequences and checked their similarity by comparing values of average nucleotide identity (ANI), calculated using a OrthoANI [32]. The genome information of several Arthrobacter species is available in GenBank, and we compared the CAZymes from registered species referenced in CAZy (http://www.cazy.org). Based on complete genome sequence, we found 25 strains from a same species. All those sequences were downloaded from the database and all CAZymes were reannotated using the dbCAN2 server.

\section{Abbreviations}

GH: Glycoside Hydrolase; CE: Carbohydrate Esterase; PL: Polysaccharide Lyase; GT: Glycosyltransferase; AA: Auxiliary Activities; CBM: Carbohydrate-Binding Module; ANI: Average Nucleotide Identity; NCBI: National Center for Biotechnology Information.

\section{Declarations}

\section{Acknowledgements}

Not applicable.

\section{Authors' contributions}

H. Park and T.-J. Oh designed and supervised the project. S.-R. Han and B. Kim performed the experiments; S.-R. Han, B. Kim, J.H. Jang, H. Park, and T.-J. Oh wrote the manuscript. All authors discussed the results, commented on the manuscript, and approved the manuscript.

\section{Funding}

This research was a part of the project titled "Development of potential antibiotic compounds using polar organism resources (15250103, KOPRI Grant PM20030)", funded by the Ministry of Oceans and Fisheries, Korea. In addition, this work was supported by the National Research Foundation of Korea (NRF) grant funded by the Korea government (MSIT) (NRF-2017R1A2B4012865).

\section{Availability of data and materials}

The datasets analyzed during the current study are available in the NCBI repository, accession numbers: NZ_CP039290.1for Arthrobacter sp. strain PAMC25564, complete genome; NZ_CP040018.1 for Arthrobactersp. strain 24S4-2, complete genome; NZ_CP022436.1 for Arthrobacter sp. strain YN, complete genome; NZ_CP019304.1 for Arthrobactersp. strain QXT-31, complete genome; NZ_CP003203.1 for Arthrobacter sp. strain Rue61a; NZ_CP000454.1 for Arthrobactersp. strain FB24, complete genome; NZ_CP007595.1 for Arthrobacter sp. strain PAMC25486, complete genome; NZ_CP017421.1 for Arthrobacter sp. strain ZXY-2, complete genome; NZ_CP015732.1 for Arthrobacter sp. strain U41, complete genome; NZ_CP029642.1 for Arthrobacter sp. strain DCT-5, complete genome; NZ_CP026514.1 for Arthrobacter sp. strain PGP41, complete genome; NZ_CP012479.1 for Arthrobacter sp. strain ERGS1:01, complete genome; NZ_CP013297.1 for Arthrobacter sp. strain YC-RL1 , complete genome; AP014718.1 for Arthrobacter sp. strain Hiyo4, complete genome; NZ_CP042172.1 for Arthrobacter sp. strain KBS0702, complete genome; NZ_CP040174.1 for Arthrobacter sp. strain UKPF54-2, complete 
genome; AP018697.1 for Arthrobacter sp. strain MN05-02, complete genome; AP014719.1 for Arthrobacter sp. strain Hiyo8, complete genome; NZ_CP014196.1 for Arthrobacter sp. strain ATCC21022, complete genome. NZ_CP018863.1 for Arthrobacter crystallopoietes DSM 20117, complete genome; NZ_CP012677.1 for Arthrobacter alpinus strain R3.8, complete genome; NZ_CP013200.1 for Arthrobacter alpinus strain ERGS4:06, complete genome; NZ_CP013745.1 for Arthrobacter alpinus strain A3, complete genome; CP002379.1 for Pseudarthrobacter phenanthrenivorans strain Sphe3, complete genome; CP001341.1 for Pseudarthrobacter chlorophenolicus strain A6, complete genome; NZ_CP013747.1 for Pseudarthrobacter sulfonivorans strain Ar51, complete genome.

\section{Ethics approval and consent to participate}

Not applicable.

\section{Consent for publication}

Not applicable.

\section{Competing interests}

The authors have declared that no conflict of interest exists.

\section{Author details}

${ }^{1}$ Department of Life Science and Biochemical Engineering, Graduate School, SunMoon University, 70 Sunmoon-ro 221, Tangjeong-myeon, Asan-si, Chungnam 31460, Republic of Korea. ${ }^{2}$ Department of Dental Hygiene, College of Health Science, Dankook University, 119 Dandae-ro, Dongnam-gu, Cheonansi, Chungnam 31116, Republic of Korea. ${ }^{3}$ Division of Biotechnology, College of Life Science and Biotechnology, Korea University, Seoul 02841, Republic of Korea. ${ }^{4}$ Genome-based BiolT Convergence Institute, 70 Sunmoon-ro 221, Tangjeong-myeon, Asan-si, Chungnam 31460, Republic of Korea. ${ }^{5}$ Department of Pharmaceutical Engineering and Biotechnology, SunMoon University, 70 Sunmoon-ro 221, Tangjeong-myeon, Asan-si, Chungnam 31460, Republic of Korea

*Co-corresponding authors: Hyun Park \& Tae-Jin Oh

H. Park, Division of Biotechnology, College of Life Sciences and Biotechnology, Korea University, Seoul 02841, Korea. Tel: +82 2 3290 3051 ; E-mail: hpark@korea.ac.kr

T.-J. Oh, Department of Pharmaceutical Engineering and Biotechnology, SunMoon University, Asan 31460, Korea. Tel: +82 41 5302677 ; E-mail: tjoh3782@sunmoon.ac.kr

\section{References}

1. J. Conn, I. Dimmick, Soil bacteria similar in morphology to Mycobacterium and Corynebacterium, J. Bacteriol. 1947; 54: $291-303$. https://doi.org/10.1128/JB.54.3.291-303.1947.

2. Koch, P. Schumann, E. Stackebrandt, Reclassification of Micrococcus agilis Ali-Cohen 1889; to the genus Arthrobacter as Arthrobacter agilis comb. nov. and emendation of the genus Arthrobacter, Int. J. Syst. Bacteriol. 1995; 45: 837-839. https://doi.org/10.1099/00207713-45-4-837.

3. Unell, K. Nordin, C. Jernberg, J. Stenstrom, J.K. Jansson, Degradation of mixtures of phenolic compounds by Arthrobacter chlorophenolicus A6, Biodegradation 2008; 19: 495-505. http://10.1007/s10532-007-9154-2.

4. G. Dastager, Q. Liu, S.K. Tang, S. Krishnamurthi, J.C. Lee, W.J. Li, Arthrobacter enclensis sp. nov., isolated from sediment sample, Arch. Microbiol. 2014; 196: 775-782. https://doi.org/10.1007/s00203-014-1016-9.

5. Huang, N. Zhao, L. He, L. Wang, Z. Liu, M. You, F. Guan, Arthrobacter scleromae sp. nov., isolated from human clinical specimens, J. Clin. Microbiol. 2005; 43: 1451-1455. https://doi.org/10.1128/JCM.43.3.1451-1455.2005.

6. W. Chang, J.W. Bae, Y.D. Nam, H.Y. Kwon, J.R. Park, K.S. Shin, K.H. Kim, Z.X. Quan, S.K. Rhee, K.G. An, Y.H. Park, Arthrobacter subterraneus sp. nov., isolated from deep subsurface water of the South Coast of Korea, J. Microbiol. Biotechnol. 2007; 17: 1875-1879.

7. Margesin, P. Schumann, D.C. Zhang, M. Redzic, Y.G. Zhou, H.C. Liu, F. Schinner, Arthrobacter cryoconiti sp. nov., a psychrophilic bacterium isolated from alpine glacier cryoconite, Int. J. Syst. Evol. Microbiol. 2012; 62: 397-402. https://doi.org/10.1099/ijs.0.031138-0.

8. K. Kim, K.C. Lee, H.M. Oh, M.J. Kim, M.K. Eom, J.S. Lee, Arthrobacter defluvii sp. nov., 4-chlorophenol-degrading bacteria isolated from sewage, Int. J. Syst. Evol. Microbiol. 2008; 58: 1916-1921. https://doi.org/10.1099/ijs.0.65550-0.

9. Liu, Y.H. Xin, X.L. Chen, H.C. Liu, Y.G. Zhou, W.X. Chen, Arthrobacter ruber sp. nov., isolated from glacier ice, Int. J. Syst. Evol. Microbiol. 2018; 68: 16161621. https://doi.org/10.1099/ijsem.0.002719.

10. Cavicchioli, Cold-adapted archaea, Nat. Rev. Microbiol. 2006; 4: 331-343.https://doi.org/10.1038/nrmicro1390.

11. Y. Morita, Psychrophilic bacteria, Bacteriol. Rev. 1975; 39: 144-167.

12. D’Amico, T. Collins, J.C. Marx, G. Feller, C. Gerday, Psychrophilic microorganisms: challenges for life, EMBO Rep. 2006 ; 7: 385-389. https://doi.org/10.1038/sj.embor.7400662.

13. Shivaji, J.S. Prakash, How do bacteria sense and respond to low temperature? Arch. Microbiol. 2010; 192: 85-95. https://doi.org/10.1007/s00203-0090539-y. 
14. Gerday, M. Aittaleb, M. Bentahir, J.P. Chessa, P. Claverie, T. Collins, S. D’Amico, J. Dumont, G. Garsoux, D. Georlette, A. Hoyoux, T. Lonhienne, M.A. Meuwis, G. Feller, Cold-adapted enzymes: from fundamentals to biotechnology, Trends Biotechnol. 2000; 18: 103-107. https://doi.org/10.1016/s0167-

7799(99)01413-4.

15. Rytioja, K. Hildén, J. Yuzon, A. Hatakka, R.P. de Vries, M.R. Mäkelä, Plant-polysaccharide-degrading enzymes from Basidiomycetes, Microbiol. Mol. Biol. Rev. 2014; 78: 614-649. https://doi.org/10.1128/MMBR.00035-14.

16. K. Sista Kameshwar, W. Qin, Comparative study of genome-wide plant biomass-degrading CAZymes in white rot, brown rot and soft rot fungi, Mycology 2017; 9: 93-105. https://doi.org/10.1080/21501203.2017.1419296.

17. Klotz, J. Georg, L. Bucinská, S. Watanabe, V. Reimann, W. Januszewski, R. Sobotka, D. Jendrossek, W.R. Hess, K. Forchhammer, Awakening of a dormant cyanobacterium from nitrogen chlorosis reveals a genetically determined program, Curr. Biol. 2016; 26: 2862-2872. https://doi.org/10.1016/j.cub.2016.08.054.

18. Klotz, K. Forchhammer, Glycogen, a major player for bacterial survival and awakening from dormancy, Future Microbiol. $2017 ; 12$ : $101-104$. https://doi.org/ 10.2217/fmb-2016-0218.

19. R. de Souza, Microbial Degradation of Lignocellulosic Biomass, In: Chandel DA (ed), InTech. 2013; pp. 207-247.

20. Valk, W. Eeuwema, F.D. Sarian, R.M. van der Kaaij, L. Dijkhuizen, Degradation of granular starch by the bacterium Microbacterium aurum strain B8. A involves a modular a-amylase enzyme system with FNIII and CBM25 domains, Appl. Environ. Microbiol. 2015; 81: 6610-6620. https://doi.org/10.1128/AEM.01029-15.

21. J. Goh, T.R. Klaenhammer, Insights into glycogen metabolism in Lactobacillus acidophilus: impact on carbohydrate metabolism, stress tolerance and gut retention, Microb. Cell Fact. 2014; 13: 94. https://doi.org/10.1186/s12934-014-0094-3.

22. S. Chin, D.H. Alexander, P. Marks, A.A. Klammer, J. Drake, C. Heiner, A. Clum, A. Copeland, J. Huddleston, E.E. Eichler, S.W. Turner, J. Korlach, Nonhybrid, finished microbial genome assemblies from long-read SMRT sequencing data, Nat. Methods 2013; 10: 563-569. https://doi.org/10.1038/nmeth.2474.

23. K. Aziz, D. Bartels, A.A. Best, M. DeJongh, T. Disz, R.A. Edwards, K. Formsma, S. Gerdes, E.M. Glass, M. Kubal, F. Meyer, G.J. Olsen, R. Olson, A.L. Osterman, R.A. Overbeek, L.K. McNeil, D. Paarmann, T. Paczian, B. Parrello, G.D. Pusch, C. Reich, R. Stevens, O. Vassieva, V. Vonstein, A. Wilke, O. Zagnitko, The RAST Server: rapid annotations using subsystems technology, BMC Genomics 2008; 9: 75. http://10.1186/1471-2164-9-75.

24. Huerta-Cepas, D. Szklarczyk, K. Forslund, H. Cook, D. Heller, M.C. Walter, T. Rattei, D.R. Mende, S. Sunagawa, M. Kuhn, L.J. Jensen, C. von Mering, P. Bork, eggNOG 4.5: a hierarchical orthology framework with improved functional annotations for eukaryotic, prokaryotic and viral sequences, Nucleic Acids Res. 2016; 44: D286-D293. https://doi.org/10.1093/nar/gkv1248.

25. R. Grant, A.S. Arantes, P. Stothard, Comparing thousands of circular genomes using the CGView Comparison Tool, BMC Genomics $2012 ; 13: 202$. https://doi.org/10.1186/1471-2164-13-202.

26. Zhang, T. Yohe, L. Huang, S. Entwistle, P. Wu, Z. Yang, P.K. Busk, Y. Xu, Y. Yin, dbCAN2: a meta server for automated carbohydrate-active enzyme annotation, Nucleic Acids Res. 2018; 46: W95-W101. https://doi.org/10.1093/nar/gky418.

27. Buchfink, C. Xie, D.H. Huson, Fast and sensitive protein alignment using DIAMOND, Nat. Methods 2015; 12: 59-60. https://doi.org/10.1038/nmeth.3176.

28. K. Busk, B. Pilgaard, M.J. Lezyk, A.S. Meyer, L. Lange, Homology to peptide pattern for annotation of carbohydrate-active enzymes and prediction of function, BMC Bioinformatics 2017; 18: 214. https://doi.org/10.1186/s12859-017-1625-9.

29. C. Edgar, MUSCLE: multiple sequence alignment with high accuracy and high throughput, Nucleic Acids Res. 2004; 32: $1792-1797$. https://doi.org/10.1093/nar/gkh340.

30. C. Edgar, MUSCLE: a multiple sequence alignment method with reduced time and space complexity, BMC Bioinformatics $2004 ; 5: 113$. https://doi.org/10.1186/1471-2105-5-113.

31. Kumar, G. Stecher, M. Li, C. Knyaz, K. Tamura, MEGA X: Molecular evolutionary genetics analysis across computing platforms, Mol. Biol. Evol. 2018; 35 : 1547-1549. https://doi.org/10.1093/molbev/msy096.

32. Lee, Y.O. Kim, S.C. Park, J. Chun, OrthoANI: an improved algorithm and software for calculating average nucleotide identity, Int. J. Syst. Evol. Microbiol. 2016; 66: 1100-1103. https://doi.org/10.1099/ijsem.0.000760.

33. L. Nelson, M.M. Cox, A.L. Lehninger, Lehninger principles of biochemistry 6 th (ed), W. H. Freeman \& Co., New York 2013.

34. M. Sanders, Carbohydrate: Digestion, absorption and metabolism. Encyclopedia of food and health, 2016; pp. 643-650.

35. J. Busse, P. Schumann, Reclassification of Arthrobacter enclensis as Pseudarthrobacter enclensis comb. nov., and emended descriptions of the genus Pseudarthrobacter, and the species Pseudarthrobacter phenanthrenivorans and Pseudarthrobacter scleromae, Int. J. Syst. Evol. Microbiol. 2019; 69: 3508-3511. https://doi.org/10.1099/ijsem.0.003652.

36. J. Busse, Review of the taxonomy of the genus Arthrobacter, emendation of the genus Arthrobacter sensu lato, proposal to reclassify selected species of the genus Arthrobacter in the novel genera Glutamicibacter gen. nov., Paeniglutamicibacter gen. nov., Pseudoglutamicibacter gen. nov., Paenarthrobacter gen. nov. and Pseudarthrobacter gen. nov., and emended description of Arthrobacter roseus, Int. J. Syst. Evol. Microbiol. 2016; 66: 9-37. https://doi.org/10.1099/ijsem.0.000702.

37. Goris, K.T. Konstantinidis, J.A. Klappenbach, T. Coenye, P. Vandamme, J.M. Tiedje, DNA-DNA hybridization values and their relationship to whole-genome sequence similarities, Int. J. Syst. Evol. Microbiol. 2007; 57: 81-91. https://doi.org/10.1099/ijs.0.64483-0.

38. Jain, L.M. Rodriguez-R, A.M. Phillippy, K.T. Konstantinidis, S. Aluru, High throughput ANI analysis of $90 \mathrm{~K}$ prokaryotic genomes reveals clear species boundaries, Nat. Commun. 2018; 9: 5114. https://doi.org/10.1038/s41467-018-07641-9. 
39. Yeo, K. Chater, The interplay of glycogen metabolism and differentiation provides an insight into the developmental biology of Streptomyces coelicolor, Microbiology 2005; 151: 855-861. https://doi.org/10.1099/mic.0.27428-0.

40. Schneider, C.J. Bruton, K.F. Chater, Duplicated gene clusters suggest an interplay of glycogen and trehalose metabolism during sequential stages of aerial mycelium development in Streptomyces coelicolor A32, Mol. Gen. Genet. 2000; 263: 543-553. https://doi.org/10.1007/s004380051200.

41. H.D. Nguyen, S.H. Park, P.L. Tran, J.W. Kim, Q.T. Le, W. Boos, J.T. Park, Characterization of the transglycosylation reaction of 4-a-glucanotransferase MalQ; and its role in glycogen breakdown in Escherichia coli, J. Microbiol. Biotechnol. 2019; 29: 357-366. https://doi.org/10.4014/jmb.1811.11051.

42. Ball, C. Colleoni, U. Cenci, J.N. Raj, C. Tirtiaux, The evolution of glycogen and starch metabolism in eukaryotes gives molecular clues to understand the establishment of plastid endosymbiosis, J. Exp. Bot. 2011; 62: 1775-1801. https://doi.org/10.1093/jxb/erq411.

43. Wang, M.J. Wise, Glycogen with short average chain length enhances bacterial durability, Naturwissenschaften 2011; 98: 719-729. https://doi.org/10.1007/s00114-011-0832-x.

44. Wang, Q. Liu, X. Tan, T. Yang, D. Tang, W. Wang, M.J. Wise, Bacterial glycogen as a durable energy reserve contributing to persistence: an updated bibliography and mathematical model, bioRxiv 2019. https://doi.org/doi.org/10.1101/536110.

45. Dippel, W. Boos, The maltodextrin system of Escherichia coli: metabolism and transport, J. Bacteriol. 2005; 187: 8322-8331. https://10.1128/JB.187.24.8322-8331.2005.

46. H. Park, Roles of enzymes in glycogen metabolism and degradation in Escherichia coli, J. Appl. Glycosci. 2015; 62: 37-45. https://10.5458/jag.jag.JAG2015_005.

47. Bornemann, a-Glucan biosynthesis and the GlgE pathway in Mycobacterium tuberculosis, Biochem. Soc. Trans. 2016; $44: 68-73$. https://10.1042/BST20150181.

48. T. Park, J.H. Shim, P.L. Tran, I.H. Hong, H.U. Yong, E.F. Oktavina, H.D. Nguyen, J.W. Kim, T.S. Lee, S.H. Park, W. Boos, K.H. Park, Role of maltose enzymes in glycogen synthesis by Escherichia coli, J. Bacteriol. 2011; 193: 2517-2526. https://doi.org/10.1128/JB.01238-10.

49. Dalmasso, J. Aubert, S. Even, H. Falentin, M.-B. Maillard, S. Parayre, V. Loux, J. Tanskanen, A. Thierry, Accumulation of intracellular glycogen and trehalose by Propionibacterium freudenreichii under conditions mimicking cheese ripening in the cold, Appl. Environ. Microbiol. $2012 ; 78: 6357-6364$. https://doi.org/10.1128/AEM.00561-12.

50. Reina-Bueno, M. Argandoña, J.J. Nieto, A. Hidalgo-Garcia, F. Iglesias-Guerra, M.J. Delgado, C. Vargas, Role of trehalose in heat and desiccation tolerance in the soil bacterium Rhizobium etli, BMC Microbiol. 2012; 12: 207. https://10.1186/1471-2180-12-207.

51. A.L. De Smet, A. Weston, I.N. Brown, D.B. Young, B.D. Robertson, Three pathways for trehalose biosynthesis in Mycobacteria, Microbiology 2000; 146: 199-208. https://doi.org/10.1099/00221287-146-1-199.

52. Wolf, R. Kramer, S. Morbach, Three pathways for trehalose metabolism in Corynebacterium glutamicum ATCC13032 and their significance in response to osmotic stress, Mol. Microbiol. 2003; 49: 1119-1134. https://10.1046/j.1365-2958.2003.03625.x.

53. Carpinelli, R. Kramer, E. Agosin, Metabolic engineering of Corynebacterium glutamicum for trehalose overproduction: role of the TreYZ trehalose biosynthetic pathway, Appl. Environ. Microbiol. 2006; 72: 1949-1955. https://doi.org/10.1128/AEM.72.3.1949-1955.2006.

54. Padilla, S. Morbach, R. Kramer, E. Agosin, Impact of heterologous expression of E. coli UDP-glucose pyrophosphorylase on trehalose and glycogen synthesis in Corynebacterium gluticum, Appl. Environ. Microbiol. 2004; 70: 3845-3854. https://doi.org/10.1128/AEM.70.7.3845-3854.2004.

55. Niewerth, J. Schuldes, K. Parschat, P. Kiefer, J.A. Vorholt, R. Daniel, S. Fetzner, Complete genome sequence and metabolic potential of the quinaldinedegrading bacterium Arthrobacter sp. Rue61a, BMC Genomics 2012; 13: 534. https://doi.org/10.1186/1471-2164-13-534.

56. H. Nakatsu, R. Barabote, S. Thompson, D. Bruce, C. Detter, T. Brettin, C. Han, F. Beasley, W. Chen, A. Konopka, G. Xie, Complete genome sequence of Arthrobactersp. strain FB24, Stand. Genomic Sci. 2013; 9: 106-116. https://doi.org/10.4056/sigs.4438185.

57. Kumar, D. Singh, M.K. Swarnkar, A.K. Singh, S. Kumar, Complete genome sequence of Arthrobactersp. ERGS1: 01, a putative novel bacterium with prospective cold active industrial enzymes, isolated from East Rathong glacier in India, J. Biotechnol. 2015; 214: $139-140$. https://doi.org/10.1016/j.jbiotec.2015.09.025.

58. Ren, Y. Jia, N. Ruth, B. Zhao, Y. Yan, Complete genome sequence of an aromatic compound degrader Arthrobacter sp. YC-RL1, J. Biotechnol. 2016; 219: 34-35. https://doi.org/10.1016/j.jbiotec.2015.12.008.

59. Hiraoka, A. Machiyama, M. Ijichi, K. Inoue, K. Oshima, M. Hattori, S. Yoshizawa, K. Kogure, W. Iwasaki, Genomic and metagenomic analysis of microbes in a soil environment affected by the 2011 Great East Japan Earthquake tsunami, BMC Genomics 2016; 17: 53. https://10.1186/s12864-016-2380-4.

60. A. Russell, G.F. Hatfull, Complete genome sequence of Arthrobacter sp. ATCC 21022, a host for bacteriophage discovery, Genome Announc. 2016; 4: e00168-16. https://doi.org/10.1128/genomeA.00168-16.

61. Kumar, D. Singh, M.K. Swarnkar, A.K. Singh, S. Kumar, Complete genome sequence of Arthrobacter alpinus ERGS4: 06, a yellow pigmented bacterium tolerant to cold and radiations isolated from Sikkim Himalaya, J. Biotechnol. 2016; 220: 86-87. https://doi.org/10.1016/j.jbiotec.2016.01.016.

62. Kallimanis, K.M. LaButti, A. Lapidus, A. Clum, A. Lykidis, K. Mavromatis, I. Pagani, K. Liolios, N. Ivanova, L. Goodwin, S. Pitluck, A. Chen, K. Palaniappan, V. Markowitz, J. Bristow, A.D. Velentzas, A. Perisynakis, C.C. Ouzounis, N.C. Kyrpides, A.I. Koukkou, C. Drainas, Complete genome sequence of Arthrobacter phenanthrenivorans type strain Sphe3, Stand. Genomic Sci. 2011; 4: 123-130. https://doi.org/10.4056/sigs.1393494.

63. A. Miranda-Ríos, J.A. Ramirez-Trujillo, B. Nova-Franco, L.F. Lozano-Aguirre Beltrán, G. Iturriaga, R. Suárez-Rodriguez, Draft genome sequence of Arthrobacter chlorophenolicus strain Mor30.16, isolated from the bean rhizosphere, Genome Announc. 2015; 3: e00360-15. https://doi.org/10.1128/genomeA.00360-15.

64. Han SR, Kim DW, Kim B, Chi YM, Kang S, Park H, Jung SH, Lee JH, Oh TJ. Complete genome sequencing of Shigella sp. PAMC 28760: Identification of CAZyme genes and analysis of their potential role in glycogen metabolism for cold survival adaptation. Microb Pathog. $2019 ; 137: 103759$.

Page 10/17 


\section{Figures}

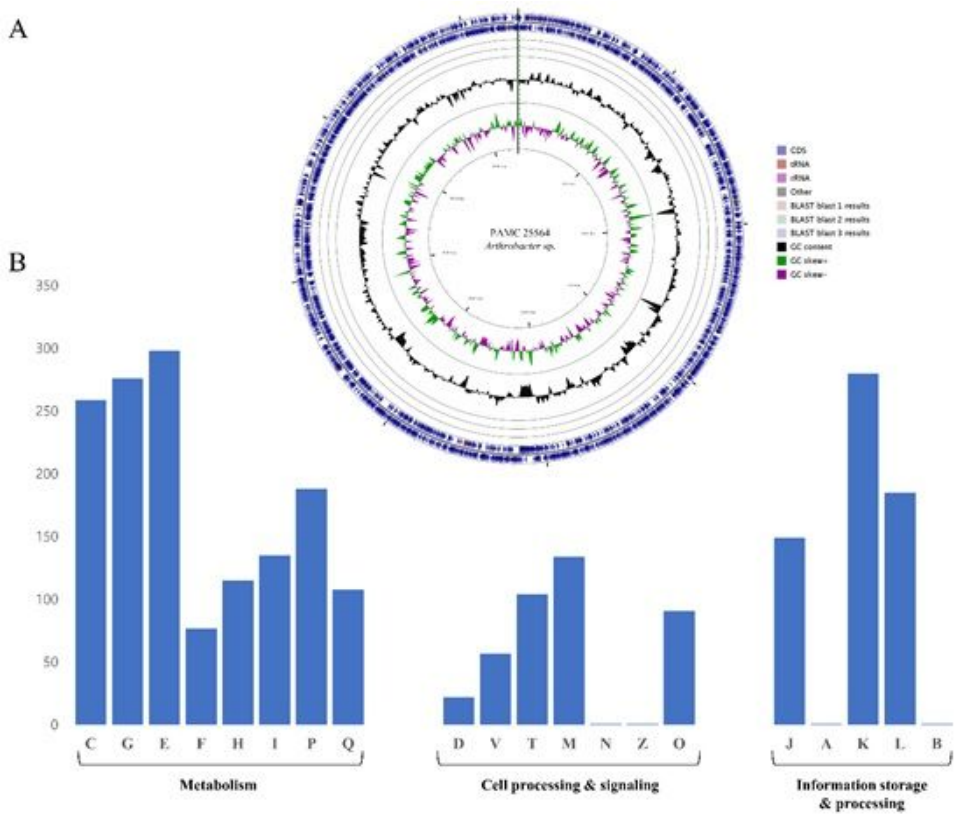

Figure 1

A; Circular map of the genome of Arthrobacter sp. PAMC25564, B; COG functional categories for forward coding sequences. Metabolism: C, energy production and conversion; $\mathrm{G}$, carbohydrate transport and metabolism; $\mathrm{E}$, amino acid transport and metabolism; F, nucleotide transport and metabolism; $\mathrm{H}$, coenzyme transport and metabolism; I, lipid transport and metabolism; P, inorganic ion transport and metabolism; and Q, secondary metabolites biosynthesis, transport, and catabolism. Cell processing and signaling: D, cell cycle control, cell division, and chromosome partitioning; $\mathrm{V}$, defense mechanisms; T, signal transduction mechanisms; M, cell wall/membrane/envelope biogenesis; N, cell motility; Z, mobilome, prophages, and transposons; and $\mathrm{O}$, posttranslational modification, protein turnover, and chaperones. Information storage and processing: J, translation, ribosomal structure, and biogenesis; A, RNA processing and modification; $\mathrm{K}$, transcription; L, replication, recombination and repair; and $\mathrm{B}$, chromatin structure and dynamics.

A

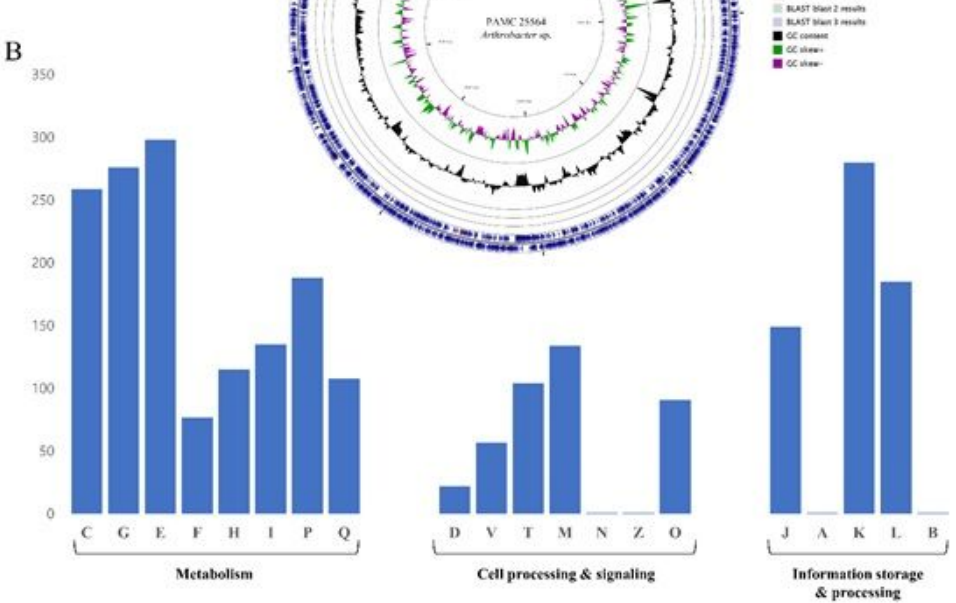

Figure 1

A; Circular map of the genome of Arthrobacter sp. PAMC25564, B; COG functional categories for forward coding sequences. Metabolism: C, energy production and conversion; $\mathrm{G}$, carbohydrate transport and metabolism; $\mathrm{E}$, amino acid transport and metabolism; $\mathrm{F}$, nucleotide transport and metabolism; $\mathrm{H}$, coenzyme transport and metabolism; I, lipid transport and metabolism; P, inorganic ion transport and metabolism; and Q, secondary metabolites biosynthesis, transport, and catabolism. Cell processing and signaling: D, cell cycle control, cell division, and chromosome partitioning; V, defense 
mechanisms; T, signal transduction mechanisms; M, cell wall/membrane/envelope biogenesis; N, cell motility; Z, mobilome, prophages, and transposons; and $\mathrm{O}$, posttranslational modification, protein turnover, and chaperones. Information storage and processing: J, translation, ribosomal structure, and biogenesis; A, RNA processing and modification; $\mathrm{K}$, transcription; $\mathrm{L}$, replication, recombination and repair; and $\mathrm{B}$, chromatin structure and dynamics.

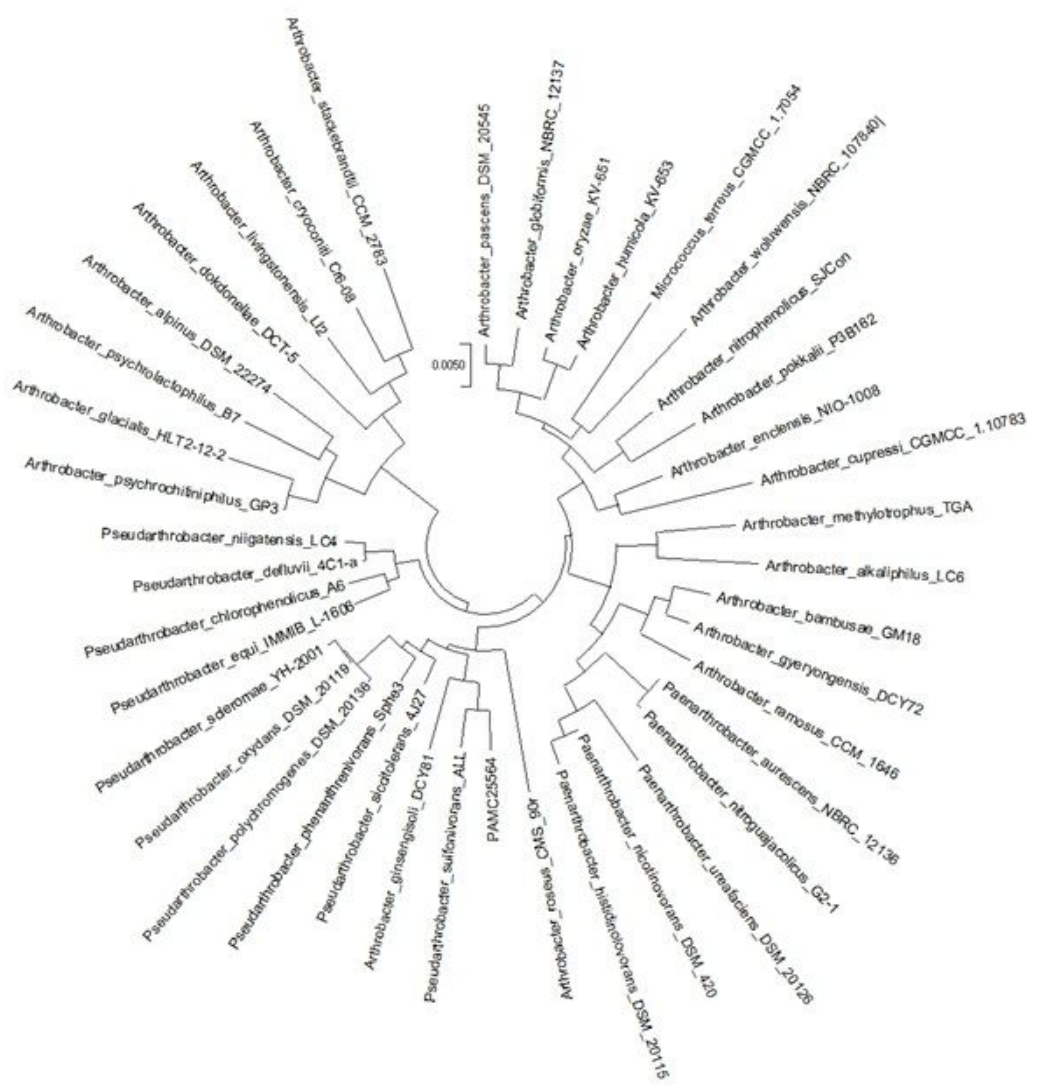

Figure 2

Phylogenetic tree of Arthrobacter sp. PAMC25564. The phylogenetic was generated using the neighbor-joining method and MEGA X, based on 16S rRNA sequences. The tree shows the relationship between three Arthrobacter strains and six Pseudarthrobacter strains, and their phylogenetic position. 


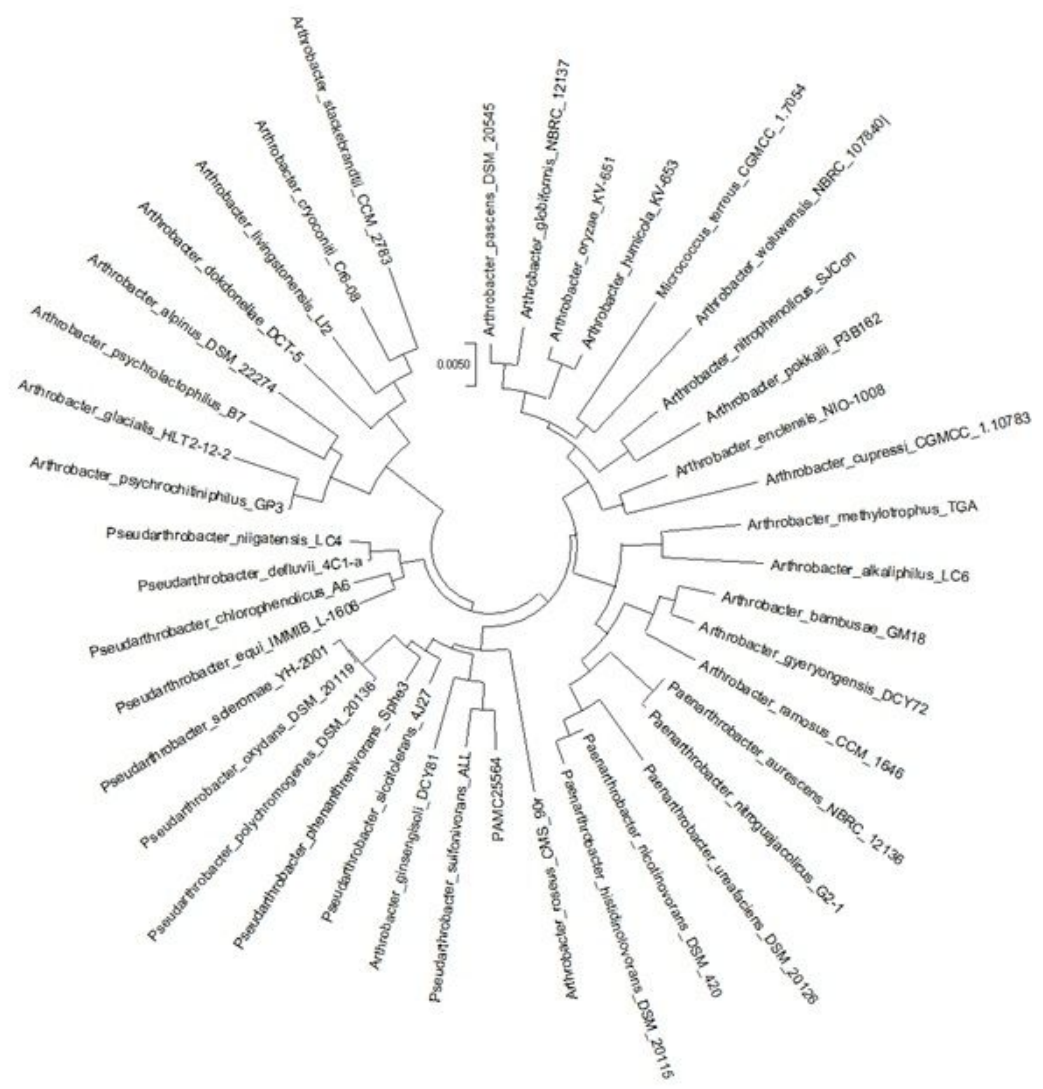

\section{Figure 2}

Phylogenetic tree of Arthrobacter sp. PAMC25564. The phylogenetic was generated using the neighbor-joining method and MEGA X, based on 16S rRNA sequences. The tree shows the relationship between three Arthrobacter strains and six Pseudarthrobacter strains, and their phylogenetic position.
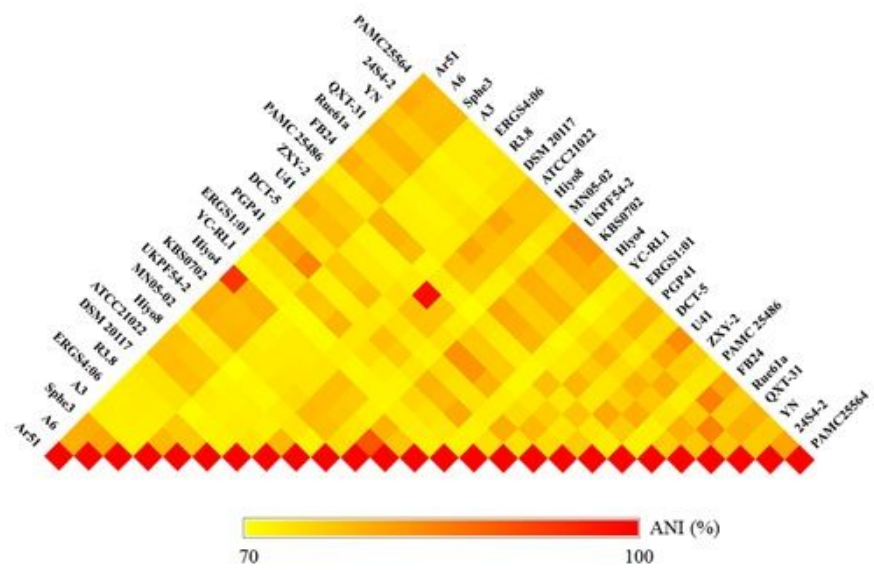

\section{Figure 3}

Average Nucleotide Identity ANl; values \%; among Arthrobacter sp. PAMC25564 genome and 25 other genomes calculated using OrthoANI. ANI results are colored yellow to red according to their value mean 70-100 \%. Strains belonging to the same species are marked with strong color. Arthrobacter sp.: PAMC25564, 24S4-2, YN, QXT-31, Rue61a, FB24, PAMC25486, ZXY-2, U41, DCT-5, PGP41, ERGS1:01, YC-RL1, Hiyo4, KBS0702, UKPF54-2, MN05-02, Hiyo8, and ATCC21022; Arthrobacter crystallopoietes: DSM 20117; Arthrobacter alpinus: R3.8, ERGS4:06, and A3; Pseudarthrobacter phenanthrenivorans: Sphe3; Pseudarthrobacter chlorophenolicus: A6; and Pseudarthrobacter sulfonivorans: Ar51. 


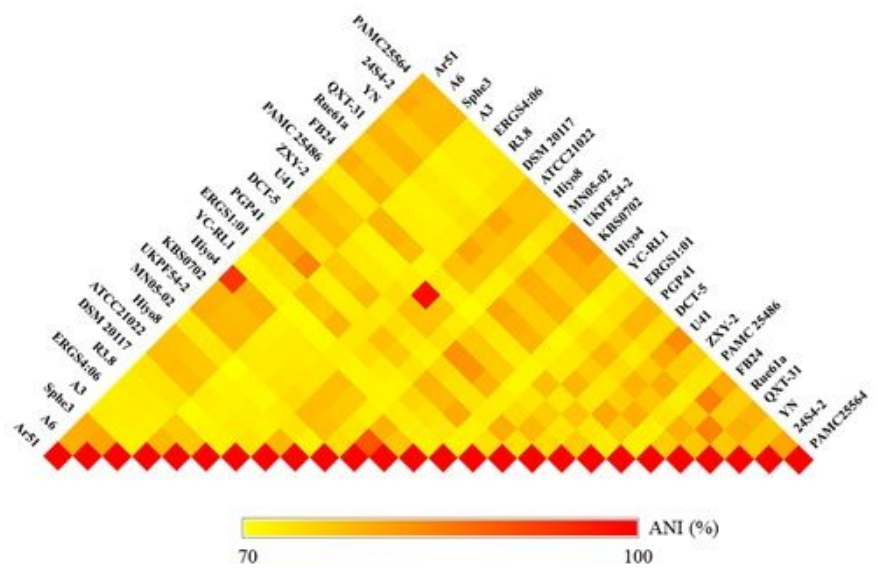

Figure 3

Average Nucleotide Identity ANI; values \%; among Arthrobacter sp. PAMC25564 genome and 25 other genomes calculated using OrthoANI. ANI results are colored yellow to red according to their value mean 70-100\%. Strains belonging to the same species are marked with strong color. Arthrobacter sp.:

PAMC25564, 24S4-2, YN, QXT-31, Rue61a, FB24, PAMC25486, ZXY-2, U41, DCT-5, PGP41, ERGS1:01, YC-RL1, Hiyo4, KBS0702, UKPF54-2, MN05-02, Hiyo8, and ATCC21022; Arthrobacter crystallopoietes: DSM 20117; Arthrobacter alpinus: R3.8, ERGS4:06, and A3; Pseudarthrobacter phenanthrenivorans: Sphe3; Pseudarthrobacter chlorophenolicus: A6; and Pseudarthrobacter sulfonivorans: Ar51.

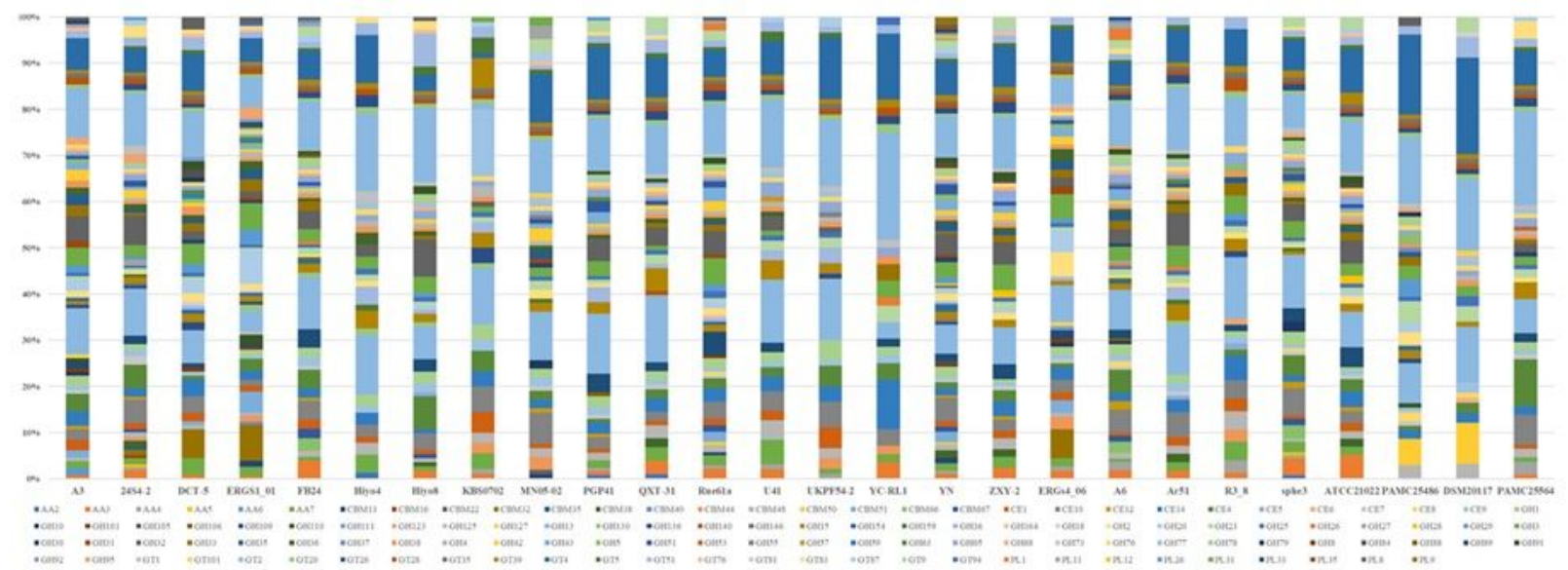

Figure 4

Comparative CAZyme-encoding genes found in the genome of Arthrobacter species. GT, glycosyl transferase; GH, glycoside hydrolase; $\mathrm{CE}$, carbohydrate esterase; CBM, carbohydrate binding; and AA, auxiliary activities. CAZyme-encoding genes are colored as indicated below the figure. Arthrobacter sp.:

PAMC25564, 24S4-2, YN, QXT-31, Rue61a, FB24, PAMC25486, ZXY-2, U41, DCT-5, PGP41, ERGS1:01, YC-RL1, Hiyo4, KBS0702, UKPF54-2, MN05-02, Hiyo8, and ATCC21022; Arthrobacter crystallopoietes: DSM 20117; Arthrobacter alpinus: R3.8, ERGS4:06, and A3; Pseudarthrobacter phenanthrenivorans: Sphe3; Pseudarthrobacter chlorophenolicus: A6; and Pseudarthrobacter sulfonivorans: Ar51.

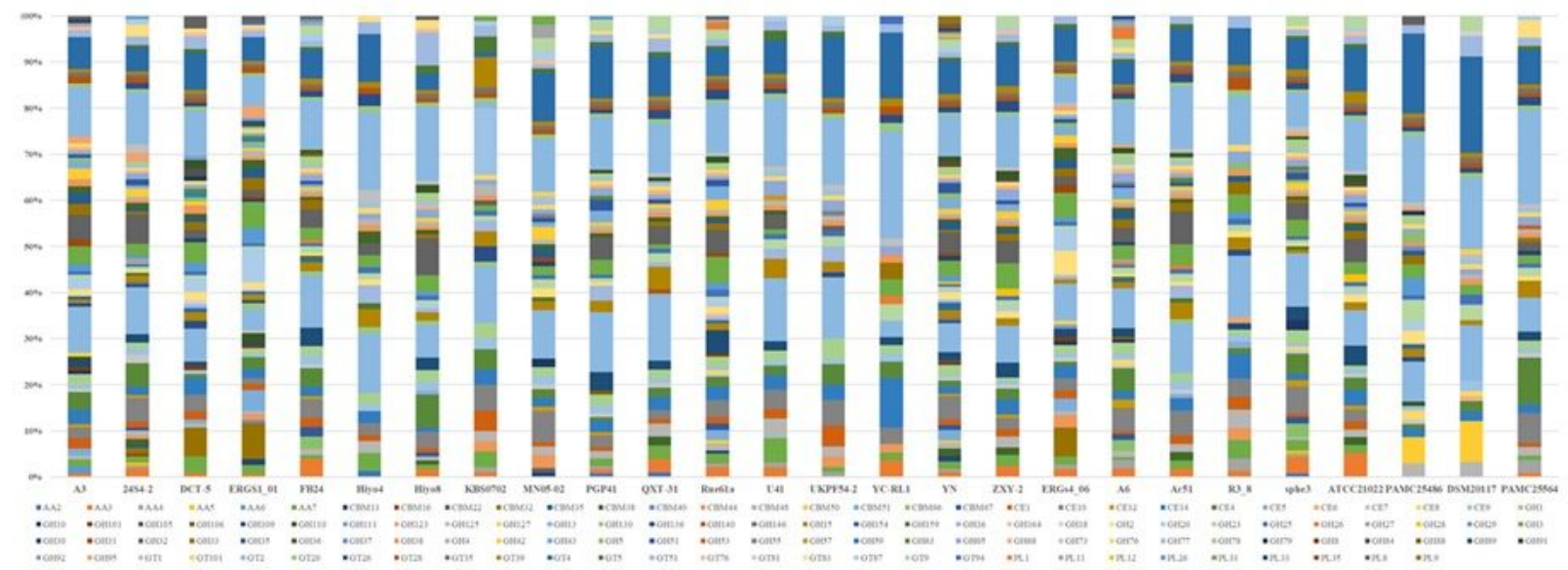

Figure 4 
Comparative CAZyme-encoding genes found in the genome of Arthrobacter species. GT, glycosyl transferase; GH, glycoside hydrolase; CE, carbohydrate esterase; CBM, carbohydrate binding; and AA, auxiliary activities. CAZyme-encoding genes are colored as indicated below the figure. Arthrobacter sp.:

PAMC25564, 24S4-2, YN, QXT-31, Rue61a, FB24, PAMC25486, ZXY-2, U41, DCT-5, PGP41, ERGS1:01, YC-RL1, Hiyo4, KBS0702, UKPF54-2, MN05-02, Hiyo8, and ATCC21022; Arthrobacter crystallopoietes: DSM 20117; Arthrobacter alpinus: R3.8, ERGS4:06, and A3; Pseudarthrobacter phenanthrenivorans: Sphe3; Pseudarthrobacter chlorophenolicus: A6; and Pseudarthrobacter sulfonivorans: Ar51.

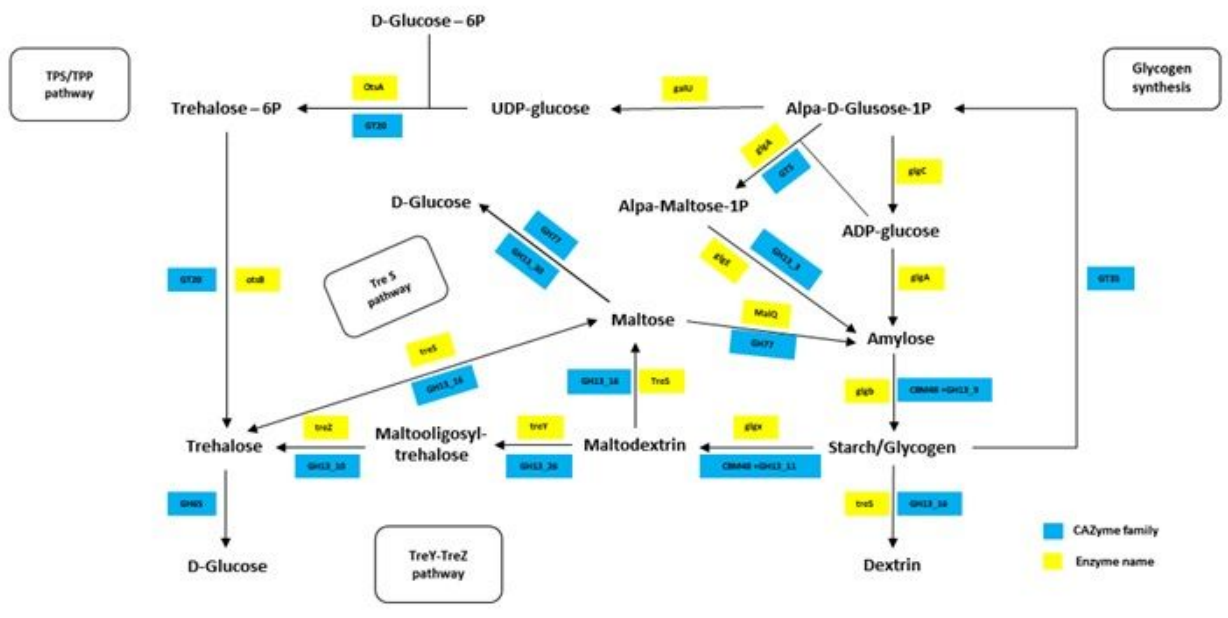

\section{Figure 5}

Predicted pathways for glycogen and trehalose metabolism in Arthrobacter sp. PAMC25564 as a response to cold adaptation. Kyoto Encyclopedia of Genes and Genomes KEGG;-predicted enzyme pathways yellow square;; and dbCAN2-predicted CAZyme family blue square;.

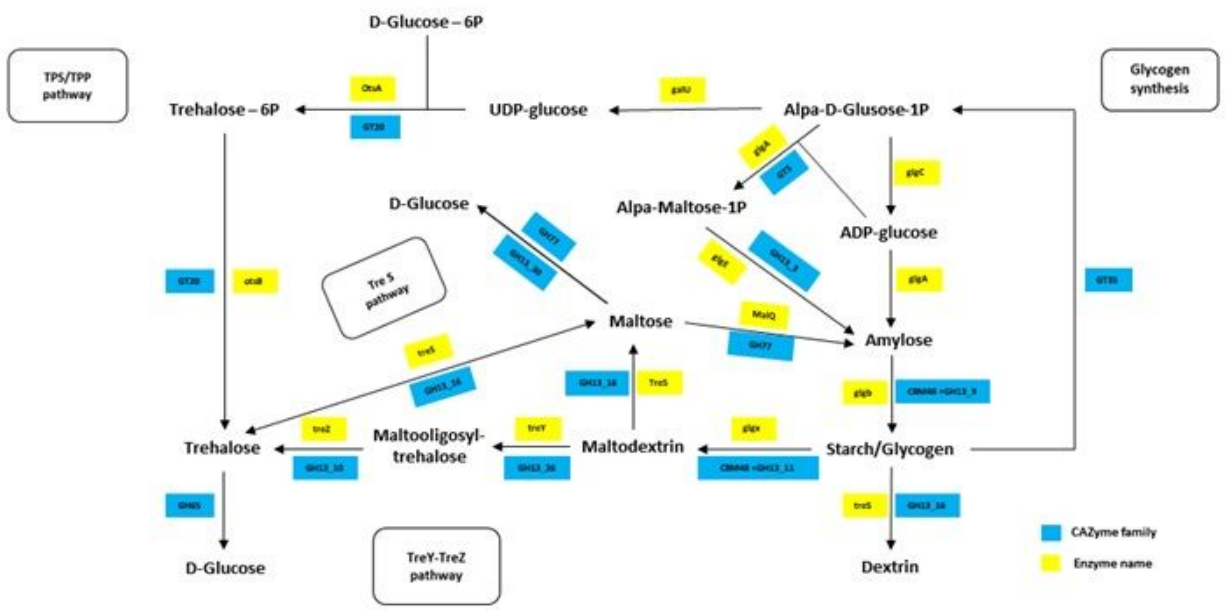

\section{Figure 5}

Predicted pathways for glycogen and trehalose metabolism in Arthrobacter sp. PAMC25564 as a response to cold adaptation. Kyoto Encyclopedia of Genes and Genomes KEGG;-predicted enzyme pathways yellow square;; and dbCAN2-predicted CAZyme family blue square;. 

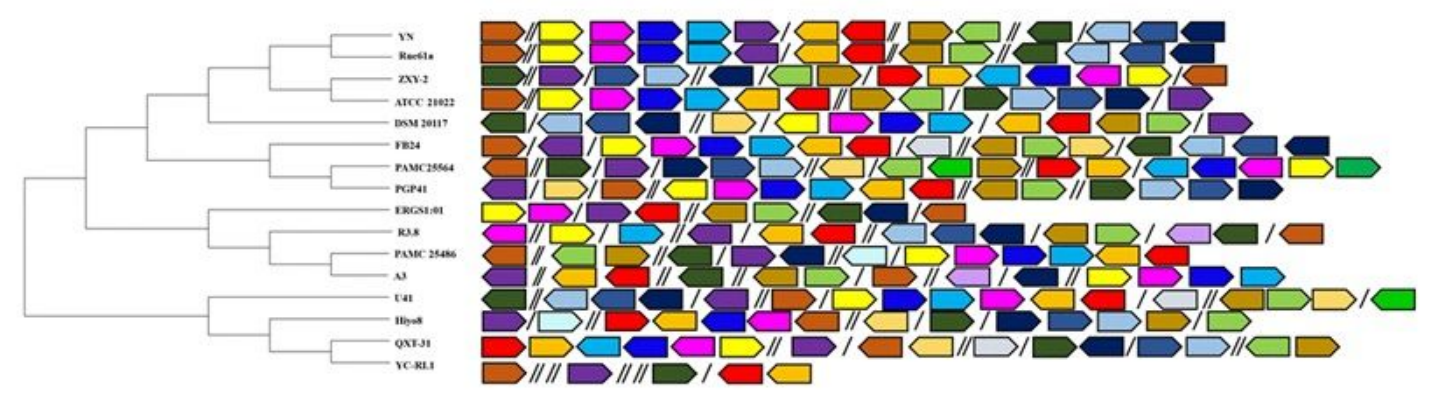

\begin{tabular}{|c|c|c|c|c|c|c|}
\hline$\square$ GH65 & 口 GH13_30 & $\square$ Tre Y & $\square$ Mal Q & $\square \mathrm{EC} 2.4 .1 .21$ & $\square \operatorname{Os} A$ & $\square^{\text {IC:32.1.28 }}$ \\
\hline & $\mathrm{x}$ & $\square^{\text {Tre Z }}$ & $\square^{\mathrm{Glg} A}$ & $\square^{\mathrm{Glg} C}$ & $\square^{\mathrm{O} s \mathrm{~B}}$ & $\square^{\mathrm{IC}}$ \\
\hline & Tre S & $\operatorname{Glg} \mathrm{E}$ & $\square$ Glg P & IC 32.1.1.196 & $\square$ EC:3.2.1.10 & \\
\hline
\end{tabular}

\section{Figure 6}

Comparative anlysis of predicted glycogen and trehalose metabolic pathways in Arthrobacter species. Glycogen and trehalose metabolism-associated gene are colored as indicated below the figure. The figure shows the differences in direction, presence, and location of genes among the strains. GH65: a,atrehalose phosphorylase; GH13_30: a-1,4-glucan-maltose-1-phosphate maltosyltransferase; TreY: 1-4;-a-D-glucan 1-a-D-glucosylmutase; MalQ: 4-aglucanotransferase; 2.4.1.21: glycogen synthase, and ADP-glucose transglucosylase; OtsA: trehalose 6-phosphate synthase; 3.2.1.28: a-trehalase; GalU: UTPglucose-1-phosphate uridylyltransferase; GlgX: glycogen debranching protein; TreZ: maltooligosyltrehalose trehalohydrolase, a-glucosidase, and a-trehalase; GIgA: glycogen synthase, and ADP-glucose transglucosylase; GlgC: glucose-1-phosphate adenylyltransferase; OtsB: trehalose 6-phosphate phosphatase; 2.7.1.201: PTS system, and sugar-specific IIA component; GlgB: 1,4-a-glucan branching enzyme; TreS: maltose a-D-glucosyltransferase/a-amylase; GlgE: a-1,4-glucan-maltose-1-phosphate maltosyltransferase, PTS system, and sugar-specific IIA component; GlgP: glycogen phosphorylase; and 3.2.1.10: oligo1,6-glucosidase.
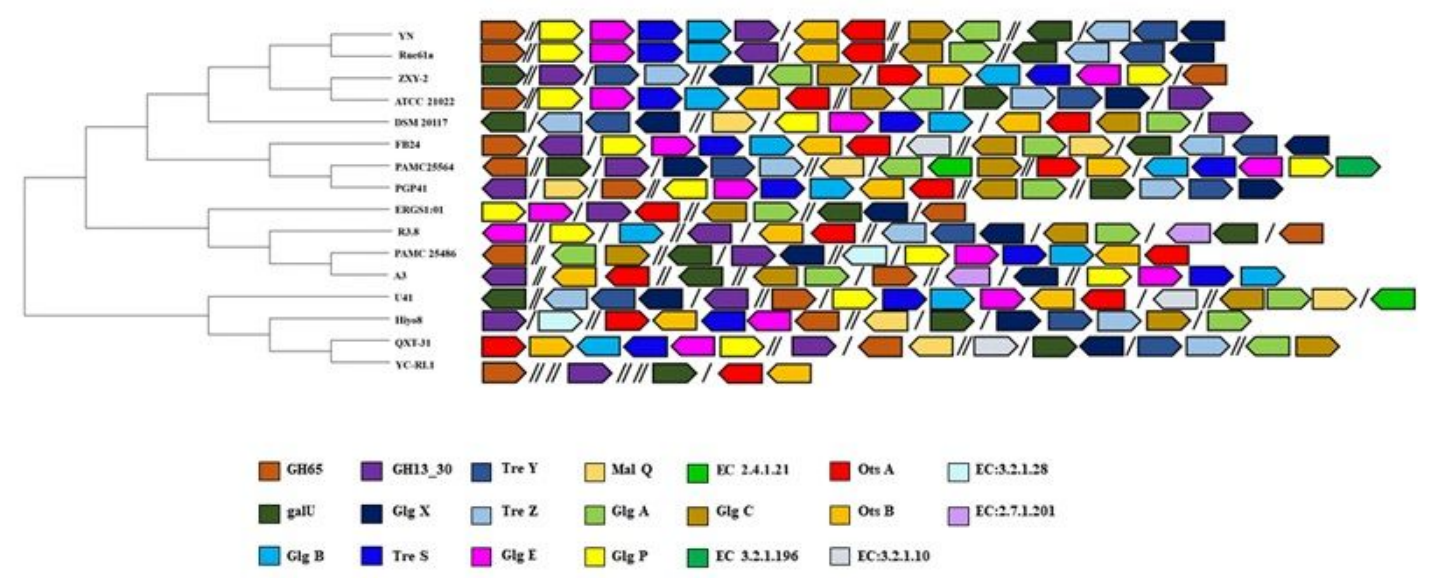

\section{Figure 6}

Comparative anlysis of predicted glycogen and trehalose metabolic pathways in Arthrobacter species. Glycogen and trehalose metabolism-associated gene are colored as indicated below the figure. The figure shows the differences in direction, presence, and location of genes among the strains. GH65: a,atrehalose phosphorylase; GH13_30: a-1,4-glucan-maltose-1-phosphate maltosyltransferase; TreY: 1 $\rightarrow$ 4;-a-D-glucan 1-a-D-glucosylmutase; MalQ: 4-aglucanotransferase; 2.4.1.21: glycogen synthase, and ADP-glucose transglucosylase; OtsA: trehalose 6-phosphate synthase; 3.2.1.28: a-trehalase; GalU: UTPglucose-1-phosphate uridylyltransferase; GlgX: glycogen debranching protein; TreZ: maltooligosyltrehalose trehalohydrolase, a-glucosidase, and a-trehalase; GlgA: glycogen synthase, and ADP-glucose transglucosylase; GlgC: glucose-1-phosphate adenylyltransferase; OtsB: trehalose 6-phosphate phosphatase; 
2.7.1.201: PTS system, and sugar-specific IIA component; GlgB: 1,4-a-glucan branching enzyme; TreS: maltose a-D-glucosyltransferase/a-amylase; GlgE: a-1,4-glucan-maltose-1-phosphate maltosyltransferase, PTS system, and sugar-specific IIA component; GlgP: glycogen phosphorylase; and 3.2.1.10: oligo1,6-glucosidase.

\section{Supplementary Files}

This is a list of supplementary files associated with this preprint. Click to download.

- Tables1.docx

- Tables1.docx 